\title{
Oportunidades de estimulação motora em ambientes domiciliares do Amazonas e fatores associados
}

\author{
Opportunities for motor stimulation in home \\ environments of Amazonas and associated factors
}

\section{Oportunidades de estimulación motora en ambientes domiciliarios de Amazonas y factores asociados}

\footnotetext{
iD (9) Marcelo Gonçalves Duarte

Universidade Federal do Mato Grosso do Sul, Corumbá, Mato Grosso do Sul, Brasil
} e-mail: duartemg83@gmail.com

iD Giseli Santos Dalpiaz Duarte

Universidade Federal do Amazonas, Parintins, Amazonas, Brasil e-mail: gisadalpiaz@gmail.com

iD Glauber Carvalho Nobre Instituto de Educação, Ciências e Tecnologia do Ceará, Fortaleza, Ceará, Brasil e-mail: glaubernobre@hotmail.com

iD Thábata Viviane Brandão Gomes Universidade Federal de Pelotas, Pelotas, Rio Grande do Sul, Brasil e-mail: thabatagomes@yahoo.com.br

iD (9) Rodolfo Novellino Benda Universidade Federal de Pelotas, Pelotas, Rio Grande do Sul, Brasil e-mail: rodolfobenda@yahoo.com.br

Resumo: O objetivo do presente estudo foi analisar as oportunidades de estimulação motora em ambientes domiciliares e fatores associados em crianças de Parintins/Amazonas. Participaram 300 pais/responsáveis por crianças da cidade de Parintins/Amazonas. Affordances in the Home Environment for Motor Development foi utilizado para avaliar oportunida- 
des de estimulação motora. A prevalência de baixa estimulação motora foi $78 \%$ maior nas casas em que moravam quatro ou mais adultos (RP: 1,78; IC95\%: 1,07-2,87); mães com o ensino fundamental (RP: 15,93; IC95\%:2,45-17,50) e ensino médio (RP: 10,07; IC95\%: 1,46-54,01) fornecem baixa estimulação motora; e crianças do sexo masculino apresentaram maior probabilidade de baixa estimulação motora (RP: 1,56; IC95\%: 1,09-1,94). Identificamos que lares nessa região não ofertam oportunidades de estimulação motora adequada para as crianças.

Palavras-chave: Crianças, oportunidades de estimulação motora, ambiente doméstico.

Abstract: The objective was to analyze the opportunities for motor stimulation of home environments and associated factors in children from Parintins/Amazonas. Participants included 300 parents/guardians of children from the city of Parintins/ Amazonas participated. Affordances in the Home Environment for Motor Development was used to assess opportunities for motor stimulation. The prevalence of low motor stimulation was $78 \%$ higher in the homes where four or more adults lived (PR: 1.78; 95\% Cl: 1.07-2.87); mothers with elementary school (PR: 15.93; 95\% Cl: 2.45-17.50) and high school (PR: 10.07; 95\% Cl: 1.46-54.01) provide low motor stimulation; and male children were more likely to have low motor stimulation (PR: 1.56; 95\% Cl: 1.09-1.94). We identified that homes in this region do not offer opportunities for adequate motor stimulation for children.

Keywords: Children, opportunities for motor stimulation, home environment

Resumen: El objetivo fue analizar las oportunidades de estimulación motora de los ambientes domésticos y factores asociados en niños de Parintins/Amazonas. Los participantes incluyeron a 300 padres/tutores de niños de la ciudad de Parintins/Amazonas. Se utilizó Affordances in the Home Environment for Motor Development para evaluar las oportunidades de estimulación motora. La prevalencia de estimulación motora baja fue $78 \%$ mayor en los hogares donde vivían cuatro o más adultos (RP: 
1,78; IC 95\%: 1,07-2,87); las madres con escuela primaria (RP: 15,93; IC del 95\%: 2,45-17,50) y secundaria (RP: 10,07; IC del 95\%: 1,46-54,01) proporcionan una estimulación motora baja; y los niños varones tenían más probabilidades de tener una estimulación motora baja (PR: 1,56; IC del 95\%: 1,09-1,94). Identificamos que los hogares en esta región no ofrecen oportunidades para la estimulación motora adecuada para los niños.

Palabras clave: Niños, oportunidades de estimulación motora, entorno familiar

Submetido em: 13-02-2021

Aceito em: 11-05-2021 
Oportunidades de estimulação motora em ambientes domiciliares do Amazonas e fatores... Marcelo Gonçalves Duarte • Giseli Santos Dalpiaz Duarte • Glauber Carvalho Nobre • Thábata Viviane Brandão Gomes • Rodolfo Novellino Benda

\section{Introdução}

O ambiente doméstico desempenha um papel vital no processo de desenvolvimento motor das crianças, principalmente nos primeiros anos de vida (FERREIRA et al., 2018; KOSHY et al., 2021; MÜLLER; VALENTINI; BANDEIRA, 2017; VALADI; GABBARD, 2020). Nesse período, as crianças permanecem a maior parte do tempo em casa e passam a experimentar diferentes formas de viver, modos de se relacionar com o mundo e ampliar suas relações com as pessoas e o meio ambiente (TOTH et al., 2020; VALADI; GABBARD, 2020; ZOGHI; SHOJAEl; GHASEMI, 2016). Um ambiente familiar favorável dá às crianças os meios para desenvolver comportamentos motores adaptativos a partir de experiências relacionadas à exploração do ambiente onde vivem, principalmente com a ação efetiva de um mediador (GABBARD; KREBS, 2012; JONES et al., 2017; TOTH et al., 2020; VALADI; GABBARD, 2020). Por outro lado, ambientes que oferecem estímulos inadequados e mediação ineficaz podem gerar dificuldades na aquisição de habilidades motoras fundamentais para o desenvolvimento infantil (FLYNN et al., 2017; NOBRE et al., 2012).

Estudos anteriores demonstraram algumas possibilidades para que esses ambientes domésticos promovam uma boa estimulação motora para crianças (BORBA; PEREIRA; VALENTINI, 2017; FERREIRA et al., 2021; PRADO; DEWEY, 2014). No Brasil, resultados de diferentes contextos do país têm reforçado essa tendência de baixa estimulação (DEFILIPO et al., 2012; FERREIRA et al., 2018; SILVA et al., 2017). Nobre et al. $(2009,2012)$ verificaram altas prevalências de inadequação de estruturas arquitetônicas e escassez de brinquedos motores finos e grossos em ambientes residenciais no estado do Ceará. Em estudos realizados em residências nos estados do Rio Grande do Sul (PILATTI et al., 2011; SOARES et al., 2015), Santa Catarina (SILVA et al., 2017), Paraná (NASCIMENTO JUNIOR et al., 2014), e Minas Gerais (DEFILIPO et al., 2012; SILVA et al., 2015), a estimulação motora deficiente também tem sido relatada, principalmente em relação aos brinquedos motores e variedade de 
Oportunidades de estimulação motora em ambientes domiciliares do Amazonas e fatores... Marcelo Gonçalves Duarte • Giseli Santos Dalpiaz Duarte • Glauber Carvalho Nobre • Thábata Viviane Brandão Gomes • Rodolfo Novellino Benda

estimulação. No Norte do Brasil, no interior do estado do Pará, observou-se estimulação insuficiente para o espaço externo e para brinquedos motores finos e grossos (ALMEIDA et al., 2015).

No que diz respeito à relação de fatores associados a uma estimulação motora deficiente, fatores sociodemográficos como estrato socioeconômico e escolaridade dos pais estiveram fortemente associados a esta conclusão (FERREIRA et al., 2018; KOSHY et al., 2021; NOBRE et al., 2012; SOARES et al., 2015). Em casas de famílias de baixo nível socioeconômico foi possível observar que existem condições inadequadas de estimulação motora, com menor oferta de brinquedos motores finos e grossos e variedade de estimulação (NOBRE et al., 2012; SOARES et al., 2015), embora os resultados a esse respeito não tenham chegado a um ponto comum na literatura. Nascimento Júnior et al. (2014), por exemplo, relataram em seu estudo que mesmo em domicílios familiares onde o nível socioeconômico era elevado, havia uma estrutura insuficiente para as oportunidades de estimulação motora para as crianças. Um segundo fator associado a essa baixa estimulação é a educação dos pais. Pais com menor escolaridade não parecem organizar seu ambiente doméstico de forma a favorecer bem a estimulação motora dos filhos (PEREIRA; VALENTINI; SACCANI, 2016; SOARES et al., 2015). Outros fatores, como quantidade de crianças e adultos por casa, tipo de residência e sexo das crianças também têm sido associados a uma baixa estimulação motora de crianças (SOARES et al., 2015).

Embora a literatura apresente um número conhecido de pesquisas sobre o tema, poucos estudos investigaram as oportunidades de estimulação motora em ambientes domiciliares no Norte do Brasil (ALMEIDA et al., 2015; DUARTE et al., 2016), em especial de crianças ribeirinhas que vivenciam algumas particularidades no seu cotidiano. Considerando a necessidade de conhecer o perfil de oportunidades de estimulação motora ofertadas nessa população, possibilitando ampliar o conhecimento sobre os fatores que influenciam nas interações entre essas crianças, a família e o ambiente onde vivem, o presente estudo objetivou analisar as 
Oportunidades de estimulação motora em ambientes domiciliares do Amazonas e fatores... Marcelo Gonçalves Duarte • Giseli Santos Dalpiaz Duarte • Glauber Carvalho Nobre • Thábata Viviane Brandão Gomes • Rodolfo Novellino Benda

oportunidades de estimulação motora de ambientes domiciliares e fatores associados em crianças de Parintins/Amazonas. Foi estabelecida a seguinte hipótese para esta investigação: os ambientes domiciliares demonstram uma baixa oportunidade de estimulação motora.

\section{Método}

\section{Características do estudo}

Esta pesquisa foi aprovada pelo Comitê de Ética em Pesquisa da Universidade Federal do Amazonas (número 860.883). A investigação foi desenvolvida na cidade de Parintins, localizada no chamado baixo Amazonas, no mesmo estado, Norte do Brasil. A população estimada de Parintins pelo Instituto Brasileiro de Geografia e Estatística (IBGE - Instituto Brasileiro de Geografia e Estatística, 2014) é de 110.441, sendo considerada a segunda cidade mais populosa do estado do Amazonas. Seu PIB (Produto Interno Bruto) é de $R \$ 673.333$ milhões em reais e o PIB per capita é de $R \$ 6.504,35$ em reais ao ano, e o IDH (Índice de Desenvolvimento Humano) de 0,658, que, segundo o PNUD (Programa das Nações Unidas para o Desenvolvimento [PNUD], 2016), é qualificado como IDH médio.

\section{Participantes}

Participaram da pesquisa 300 pais/responsáveis por crianças com idade entre 36-42 meses, destas crianças, 133 do sexo masculino $(43,3 \%)$ e 167 do sexo feminino (55,7\%), todas devidamente matriculadas em pré-escolas públicas da cidade de Parintins/ Amazonas.

\section{Instrumentos}

Este estudo aplicou o Affordances in the Home Environment for Motor Development (AHEMD) para avaliar as oportunidades de estimulação motora. O AHEMD foi desenvolvido para avaliar a quanti- 
Oportunidades de estimulação motora em ambientes domiciliares do Amazonas e fatores... Marcelo Gonçalves Duarte • Giseli Santos Dalpiaz Duarte • Glauber Carvalho Nobre • Thábata Viviane Brandão Gomes • Rodolfo Novellino Benda

dade e a qualidade das oportunidades de estimulação motora que um contexto familiar proporciona para o desenvolvimento infantil (RODRIGUES, 2005; RORIGUES; SARAIVA; GABBARD, 2005). O questionário é direcionado aos pais/responsáveis por crianças entre 36 e 42 meses e é composto por cinco subescalas: (1) espaço exterior (espaço físico externo e aparatos externos); (2) espaço interno (espaço físico interno, dispositivos internos, superfícies internas, espaço); (3) variedade de estimulação (estímulo lúdico, liberdade de movimento, estimulação e encorajamento, atividades diárias); (4) habilidades motoras finas (réplicas de brinquedos, brinquedos educativos, jogos, construção de brinquedos, materiais) e (5) materiais de corpo amplo (outros materiais musicais, materiais motores amplos, materiais de locomoção, materiais corporais). Essas subescalas são classificadas, hierarquicamente, em quatro níveis: (1) muito pobre; (2) pobre; (3) bom e (4) muito bom. A soma dos valores padronizados dessas cinco subescalas determina o valor do AHEMD total, que representa a quantidade e a qualidade das oportunidades de estimulação motora presentes no contexto familiar. O escore total do AHEMD varia de 5 a 20 pontos, que finalmente é classificado em uma escala padronizada de três categorias/níveis: 1) baixa (5 a 9 pontos); média (10 a 15 pontos) e alta (16 a 20 pontos). Neste estudo, foram utilizados os dois extremos das categorias do AHEMD: baixa e alta oportunidades de estimulação motora presentes no contexto familiar.

As variáveis independentes investigadas foram: sexo (masculino e feminino), quantidade de adultos em casa (1, 2, 3 e $\geq 4$ ), quantidade de filhos em casa (1, 2, 3 e $\geq 4$ ), escolaridade da mãe e do pai (ensino fundamental, médio e superior) e estrato socioeconômico.

\section{Procedimentos}

Primeiramente, foram contatados os diretores da pré-escola da cidade de Parintins, estado do Amazonas e, em seguida, agendados encontros com os pais e responsáveispelas crianças. Nesses encontros, as propostas de pesquisa foram expostas e os 
Oportunidades de estimulação motora em ambientes domiciliares do Amazonas e fatores... Marcelo Gonçalves Duarte • Giseli Santos Dalpiaz Duarte • Glauber Carvalho Nobre • Thábata Viviane Brandão Gomes • Rodolfo Novellino Benda

pais e responsáveis foram convidados a participar do estudo por meio de termo de consentimento assinado. O preenchimento e apresentação do protocolo AHEMD ocorreram nas pré-escolas públicas de Parintins, em locais e horários agendados pela pesquisadora. Após a aplicação do protocolo, os dados coletados foram introduzidos e classificados com o auxílio de um aplicativo do Microsoft Excel (AHEMD Calculador VPbeta1.5.xIs), construído pelos criadores do Projeto AHEMD (RODRIGUES, 2005; RORIGUES; SARAIVA; GABBARD, 2005).

\section{Análise de dados}

Os resultados foram analisados pelo Statistical Package for Social Sciences (SPSS) ${ }^{\circledR}$, versão 20.0, um software estatístico. Para analisar as variáveis associadas à estimulação motora foi utilizada a regressão de Poisson. Essa técnica se justifica uma vez que sua conclusão pode apresentar uma prevalência elevada ( $20 \%$ ou mais), o que causaria uma superestimação das razões por odds ratios (OR), obtidas por regressão logística (MAROCO, 2018). Foram calculadas as razões de prevalência (RP) brutas e ajustadas, bem como seus respectivos intervalos de confiança de 95\% (IC95\%). Para todas as análises, foi considerado um nível de significância de $5 \%$.

\section{Resultados}

A maioria dos pais $(65,7 \%)$ apresentava escolaridade até o Ensino Fundamental ( $5^{\mathrm{a}}$ a $8^{\mathrm{a}}$ série) e estrato socioeconômico classificado DE (58,9\%). A Tabela 1 mostra as frequências relativas e absolutas que caracterizam a amostra do estudo. 
Oportunidades de estimulação motora em ambientes domiciliares do Amazonas e fatores... Marcelo Gonçalves Duarte • Giseli Santos Dalpiaz Duarte • Glauber Carvalho Nobre • Thábata Viviane Brandão Gomes • Rodolfo Novellino Benda

TABELA 1. Distribuição da frequência das variáveis independentes quanto às características, estrutura familiar / domiciliar e estrato socioeconômico.

\begin{tabular}{|c|c|c|}
\hline Variáveis & Frequência relativa (\%) & Frequência absoluta (n) \\
\hline \multicolumn{3}{|l|}{ Sexo } \\
\hline Masculino & 44,3 & 133 \\
\hline Feminino & 55,7 & 167 \\
\hline \multicolumn{3}{|l|}{ Adultos em casa } \\
\hline 1 adulto & 2,3 & 07 \\
\hline 2 adultos & 8,7 & 26 \\
\hline 3 adultos & 21,3 & 64 \\
\hline$\geq 4$ adultos & 67,7 & 203 \\
\hline \multicolumn{3}{|l|}{ Crianças em casa } \\
\hline 1 criança & 7,3 & 22 \\
\hline 2 crianças & 13,0 & 39 \\
\hline 3 crianças & 43,7 & 131 \\
\hline$\geq 4$ crianças & 36,0 & 108 \\
\hline \multicolumn{3}{|l|}{ Escolaridade do pai } \\
\hline Ensino fundamental & 69,3 & 208 \\
\hline Ensino médio & 26,7 & 80 \\
\hline Superior & 4,0 & 12 \\
\hline \multicolumn{3}{|l|}{ Escolaridade da mãe } \\
\hline Ensino fundamental & 62,0 & 186 \\
\hline Ensino médio & 31,3 & 94 \\
\hline Superior & 6,7 & 20 \\
\hline \multicolumn{3}{|l|}{ Tipo de residência } \\
\hline Casa & 100,0 & 300 \\
\hline Apartamento & 0,0 & 00 \\
\hline \multicolumn{3}{|l|}{ Estrato socioeconômico } \\
\hline DE & 58,9 & 176 \\
\hline $\mathrm{C} 2$ & 41,1 & 124 \\
\hline
\end{tabular}

Fonte: Elaborada pelos autores.

Em relação à qualidade das oportunidades de estimulação motora domiciliar, 167 das crianças (55,7\%) apresentaram estimulação baixa e 48 crianças (16\%) estimulação alta. Considerando os fatores do instrumento utilizado, mas dentro e fora dos espaços, mais de $50 \%$ das crianças apresentaram estimulação muito pobre (Tabela 2). 
Oportunidades de estimulação motora em ambientes domiciliares do Amazonas e fatores... Marcelo Gonçalves Duarte • Giseli Santos Dalpiaz Duarte • Glauber Carvalho Nobre • Thábata Viviane Brandão Gomes • Rodolfo Novellino Benda

TABELA 2. Distribuição da frequência das subescalas do AHMED.

\begin{tabular}{|l|c|c|c|c|}
\hline Subescalas & $\begin{array}{c}\text { Muito pobre } \\
\text { N }(\%)\end{array}$ & $\begin{array}{c}\text { Pobre } \\
\text { N(\%) }\end{array}$ & $\begin{array}{c}\text { Bom } \\
\text { N (\%) }\end{array}$ & $\begin{array}{c}\text { Muito bom } \\
\text { N (\%) }\end{array}$ \\
\hline Espaço interno & $127(42,3)$ & $10(35,7)$ & $46(15,3)$ & $20(6,7)$ \\
\hline Espaço externo & $102(34)$ & $11(39,7)$ & $59(19,7)$ & $20(6,7)$ \\
\hline Variedade de estimulação & $226(75,3)$ & $29(9,7)$ & $2(0,7)$ & $43(14,3)$ \\
\hline Habilidades motoras finas & $220(73,3)$ & $48(16)$ & $9(3)$ & $23(7,7)$ \\
\hline $\begin{array}{l}\text { Habilidades motoras am- } \\
\text { plas }\end{array}$ & $167(55,7)$ & $85(28,3)$ & $6(2)$ & $42(14)$ \\
\hline
\end{tabular}

Fonte: Elaborada pelos autores.

Em relação à análise ajustada na regressão de Poisson, as associações foram as mesmas apenas para as variáveis: adultos em casa e escolaridade da mãe, nas quais residências com apenas um adulto (RP: 1,78; IC95\%: 1,07-2,87) a prevalência de baixa estimulação motora era $78 \%$ maior do que em casas onde moravam quatro adultos ou mais. Da mesma forma, mães que estudaram até o ensino fundamental (RP: 15,93; IC95\%:2,45-17,50) e ensino médio (RP: 10,07; IC95\%: 1,46-54,01) têm mais chances de fornecer estimulação motora insuficiente em comparação com aquelas que possuem um diploma universitário. No que diz respeito ao sexo, na análise ajustada essa variável se mostrou associada às crianças do sexo masculino, apresentando maior probabilidade de uma baixa estimulação motora em relação às crianças do sexo feminino (RP: 1,56; IC95\%: 1,09-1,94) (Tabela 3). 
Oportunidades de estimulação motora em ambientes domiciliares do Amazonas e fatores... Marcelo Gonçalves Duarte • Giseli Santos Dalpiaz Duarte • Glauber Carvalho Nobre • Thábata Viviane Brandão Gomes • Rodolfo Novellino Benda

TABELA 3. Razão de prevalência e intervalos de confiança da análise bruta e ajustada da regressão de Poisson entre a classificação da AHMED.

\begin{tabular}{|c|c|c|c|c|}
\hline \multirow[b]{3}{*}{ Fatores } & \multirow{3}{*}{$\begin{array}{c}\text { RP (IC 95\%) } \\
\text { Análise bruta }\end{array}$} & \multicolumn{2}{|c|}{ Baixa Estimulação Motora } & \multirow[b]{3}{*}{ p-valor* } \\
\hline & & & RP (IC 95\%) & \\
\hline & & p-valor* & Análise ajustada & \\
\hline \multicolumn{5}{|l|}{ Sexo } \\
\hline Masculino & $1,13(0,92-1,38)$ & 0,071 & $1,56(1,09-1,94)$ & $<0,001$ \\
\hline Feminino & 1 & & 1 & \\
\hline \multicolumn{5}{|l|}{ Adultos em casa } \\
\hline 1 adulto & $1,64(1,18-2,28)$ & 0,063 & $1,78(1,07-2,87)$ & $<0,001$ \\
\hline 2 adultos & $1,18(0,85-1,64)$ & 0,098 & $0,92(0,66-1,34)$ & 0,691 \\
\hline 3 adultos & $1,17(0,92-1,48)$ & 0,127 & $1,18(0,98-1,54)$ & 0,245 \\
\hline$\geq 4$ adultos & 1 & & 1 & \\
\hline \multicolumn{5}{|l|}{ Crianças em casa } \\
\hline 1 criança & $1,41(1,06-1,88)$ & 0,196 & $1,34(0,91-1,67)$ & 0,354 \\
\hline 2 crianças & $0,75(0,50-1,14)$ & 0,144 & $0,78(0,59-1,21)$ & 0,892 \\
\hline 3 crianças & $1,05(0,84-1,32)$ & 0,084 & $1,34(0,92-1,78)$ & 0,089 \\
\hline$\geq 4$ crianças & 1 & & 1 & \\
\hline \multicolumn{5}{|l|}{ Escolaridade do pai } \\
\hline Ensino fundamental & $3,29(0,92-11,72)$ & 0,062 & $2,19(0,57-7,62)$ & 0,261 \\
\hline Ensino médio & $3,83(1,07-13,70)$ & 0,116 & $3,332(0,83-11,76)$ & 0,832 \\
\hline Superior & 1 & & 1 & \\
\hline \multicolumn{5}{|l|}{ Escolaridade da mãe } \\
\hline Ensino fundamental & $12,47(1,84-84,54)$ & 0,065 & $15,93(2,45-17,50)$ & $<0,001$ \\
\hline Ensino médio & $10,64(1,56-72,54)$ & 0,078 & $10,07(1,46-54,01)$ & 0,021 \\
\hline Superior & 1 & & 1 & \\
\hline \multicolumn{5}{|l|}{ Tipo de residência } \\
\hline Casa & $1,35(1,02-1,97)$ & 0,386 & - & - \\
\hline Apartamento & 1 & & & \\
\hline \multicolumn{5}{|l|}{ Estrato socioeconômico } \\
\hline DE & $1,35(1,11-1,65)$ & 0,301 & - & - \\
\hline $\mathrm{C} 2$ & 1 & & & \\
\hline
\end{tabular}

Nota: categoria de referência: estimulação motora alta; $R P=$ razão de prevalência. $I C=$ intervalo de confiança. Valores em negrito são estatisticamente significativos $(p \leq 0,05)$.

Fonte: Elaborada pelos autores. 
Oportunidades de estimulação motora em ambientes domiciliares do Amazonas e fatores... Marcelo Gonçalves Duarte • Giseli Santos Dalpiaz Duarte • Glauber Carvalho Nobre • Thábata Viviane Brandão Gomes • Rodolfo Novellino Benda

\section{Discussão}

O objetivo deste estudo foi analisar as oportunidades de estimulação motora em ambientes domiciliares e fatores associados em crianças da cidade de Parintins/Amazonas. De maneira geral, as residências avaliadas no estudo apresentaram uma baixa oportunidade de estimulação motora, confirmando a hipótese estabelecida no presente estudo. A variedade de estímulos e brinquedos motores finos foram as dimensões que apresentaram os piores índices. Estudos anteriores relataram resultados negativos sobre oportunidades de estimulação motora em um ambiente doméstiCO (BUENO; CASTRO; CHIQUETTI, 2014; SILVA et al., 2015; SOARES et al., 2015; VIEIRA; SILVA; FRÔNIO, 2017). A configuração estrutural do ambiente doméstico é um fator importante a ser considerado na promoção do desenvolvimento motor das crianças, principalmente no primeiro ano de vida (FERREIRA et al. 2018; VALADI; GABBARD, 2020). Portanto, a tipologia dos espaços, considerando, por exemplo, a presença de escadas, rampas, diferentes texturas de solo e a disponibilidade de diversos brinquedos são fundamentais, uma vez que desafiam as crianças a construir comportamentos motores adaptativos a partir da exploração do ambiente (RODRIGUES; GABBARD, 2007). Consequentemente, ambientes domiciliares cujos espaços não oferecem essa possibilidade de exploração podem dificultar o processo de desenvolvimento motor, tanto em complexidade quanto em diversidade (TANI et al., 1998), como foi investigado nesta pesquisa.

Neste estudo, a presença de baixa estimulação motora em casa foi associada ao sexo. Casas com meninos demonstraram maior probabilidade de estimulação motora deficiente em comparação às casas com meninas, mesmo achado no estudo de Silva et al. (2017). Os cuidadores, que em geral são as mães, estabelecem regras específicas de acordo com o sexo dos filhos, que determinam os brinquedos a serem usados, bem como as brincadeiras usuais e a organização dos espaços da casa (GREGOVISKI; SILVA; HLAVAC, 2016). Além disso, mães de meninas podem assumir 
Oportunidades de estimulação motora em ambientes domiciliares do Amazonas e fatores... Marcelo Gonçalves Duarte • Giseli Santos Dalpiaz Duarte • Glauber Carvalho Nobre • Thábata Viviane Brandão Gomes • Rodolfo Novellino Benda

práticas parentais de monitoramento positivo e, por outro lado, mães de meninos têm apresentado comportamentos parentais mais descuidados ou desleixados (SILVA et al., 2017; RODRIGUES; NOGUEIRA; ALTAFIM, 2013). Nesse sentido, comportamentos parentais descuidados ou desleixados, assim como regras que diferenciam brinquedos e jogos podem refletir em uma má organização das oportunidades de estimulação motora no ambiente doméstico com meninos. No entanto, esses achados não significam que os meninos apresentem uma proficiência motora ruim quando comparados às meninas (NOBRE et al., 2012). Além disso, tal distinção de comportamentos de cuidadores, mães na maioria das vezes, demonstra que o processo de desenvolvimento motor não é apenas biológico, mas sim o resultado de uma interação entre aspectos maturacionais e socioculturais (BASSO; SANTOS; BENDA, 2016; GOTTLIEB, 2000; LEWONTIN, 2000).

A quantidade de adultos no domicílio também foi significativamente associada à baixa estimulação motora nos domicílios investigados. Neste estudo, lares com apenas um adulto apresentaram menor oportunidade de estimulação motora quando comparados a lares onde moravam quatro ou mais adultos. Estudos anteriores relataram resultados semelhantes (AMMAR, D.; ACEVEDO, G. A.; CORDOVA, A., 2013; BORBA; PEREIRA; VALENTINI, 2017; GIORDANI; ALMEIDA; PACHECO, 2013). A presença de mais adultos pode exigir mudanças na organização funcional e até mesmo nas estruturas da casa, com mais espaços internos, como quartos e salas; e espaços externos, como por exemplo, varandas e pátios. Possivelmente, quanto menos adultos em casa, menor será a necessidade de organização desses espaços e, portanto, menor será a disponibilidade de estimulação motora infantil no ambiente doméstico.

No presente estudo, a escolaridade da mãe foi fortemente associada a uma estimulação motora deficiente. Os lares de crianças cujas mães tinham menor escolaridade (ensino fundamental e médio) apresentaram menos oportunidades de estimulação motora quando comparados àqueles em que as mães tinham ensino superior. A condição de escolaridade tem sido um dos fatores 
Oportunidades de estimulação motora em ambientes domiciliares do Amazonas e fatores... Marcelo Gonçalves Duarte • Giseli Santos Dalpiaz Duarte • Glauber Carvalho Nobre • Thábata Viviane Brandão Gomes • Rodolfo Novellino Benda

mais associados às oportunidades de estimulação no ambiente familiar (FERREIRA et al., 2018; KOSHY et al., 2021; SOARES et al., 2015). Mães com maior escolaridade podem ter mais acesso a informações relacionadas à qualidade e organização do ambiente físico, variedade na estimulação e disponibilidade de jogos e brinquedos, de maneira que podem compreender e organizar o ambiente doméstico de forma a favorecer o desenvolvimento motor de seus filhos (FERREIRA et al., 2021; KOSHY et al., 2021; VALADI; GABBARD, 2018).

\section{Conclusão}

Em suma, as características organizacionais do ambiente nos domicílios investigados não oferecem às crianças maiores oportunidades de estimulação motora. Na maioria das residências, os espaços internos e externos e, sobretudo, a variedade de estímulos e disponibilidade de materiais são insuficientes para proporcionar a estimulação motora das crianças ribeirinhas. Além disso, fatores como menor escolaridade materna, lares com meninos e menos adultos foram associados a pouca oportunidade de estimulação motora nos domicílios investigados.

A organização de um ambiente domiciliar, assim como a meIhor qualidade e quantidade de equipamentos, brinquedos, e a disponibilização de espaços físicos mais amplos podem oportunizar às crianças condições otimizadas de explorar, vivenciar, aprender e se desenvolver. Faz-se necessário ressaltar a relevância da avaliação dos demais contextos em que a criança está inserida frequentemente, bem como o seu desenvolvimento motor, para que possam ser obtidas informações mais fidedignas e completas sobre o papel dos contextos no desenvolvimento infantil. Devese enfatizar que alguns aspectos importantes deste estudo (por exemplo, comportamentos dos pais, especialmente influências maternas e regionais na arquitetura doméstica, contexto geográfico e sociocultural) podem influenciar a organização dos espaços internos e externos, a variedade de estímulos e disponibilidade 
Oportunidades de estimulação motora em ambientes domiciliares do Amazonas e fatores... Marcelo Gonçalves Duarte • Giseli Santos Dalpiaz Duarte • Glauber Carvalho Nobre • Thábata Viviane Brandão Gomes • Rodolfo Novellino Benda

de materiais para promover o desenvolvimento motor das crianças. Uma das limitações do presente estudo é saber como esses aspectos influenciam o desenvolvimento motor. Essas limitações revelam novas questões a serem investigadas em estudos futuros.

\section{Divulgação de interesse}

Os autores informam que não há conflitos de interesse. Os autores são os únicos responsáveispelo conteúdo e redação deste artigo.

\section{Referências}

ALMEIDA, T. G. A. et al. Comparisons between motor performance and opportunities for motor stimulation in the home environment of infants from the North and Southwest regions in Brazil. Fisioterapia e pesquisa, São Paulo, v. 22, n. 2, p. 142-147, 2015. AMMAR, D.; ACEVEDO, G. A.; CORDOVA, A. Affordances in the home environment for motor development: A cross-cultural study between American and Lebanese children. Child Development Research, EUA, v. 66, n. 1, p.1-5, 2013.

BASSO, L.; SANTOS, F. G.; BENDA, R. N. Estudo do desenvolvimento motor: tendências e perspectivas. In: TANI, G. Comportamento motor: conceitos, estudos e aplicações. Rio de Janeiro, RJ: Guanabara Koogan, 2016. p. 25-30.

BORBA, L. S.; PEREIRA, K. R. G.; VALENTINI, N. C. Motor and cognitive development of infants of adolescent and adult mothers: longitudinal study. Revista Brasileira de Cineantropometria e Desempenho Humano, Florianópolis, v. 17, n. 4, p. 438-449, 2017. doi: http://dx.doi.org/10.5007/1980-0037.2015v17n4p438 BUENO, E. A.; CASTRO, A. A. M.; CHIQUETTI, E. M. S. Influência do Ambiente Domiciliar no Desenvolvimento Motor de Lactentes 
Oportunidades de estimulação motora em ambientes domiciliares do Amazonas e fatores... Marcelo Gonçalves Duarte • Giseli Santos Dalpiaz Duarte • Glauber Carvalho Nobre • Thábata Viviane Brandão Gomes • Rodolfo Novellino Benda

Nascidos Pré-Termo. Revista Neurociências, São Paulo, v. 22, n. 1, p. 45-52, 2014. doi: 10.4181/RNC.2014.22.914.8p

DEFILIPO, É. C. et al. Oportunidades do ambiente domiciliar para o desenvolvimento motor. Revista de Saúde Pública, São Paulo, v. 46, n. 4, p. 633-641, 2012.

DUARTE, M. G. et al. Desenvolvimento motor e fatores associados de crianças entre 36 e 42 meses em um contexto do baixo Amazonas. Revista da Educação Física/UEM, Maringá, v. 27, n. 4, p. 2-10, 2016.

FERREIRA, L. et al. Motor development in school-age children is associated with the home environment including socioeconomic status. Child: Care Health and Development, London, v. 44, n. 6 , p. 1-6, 2018.

FERREIRA, T. et al. Opportunities in child motor development at home: bibliometric and scientometric review. Journal Human Growth Development, Santo André, v. 31, n. 1, p. 125-144, 2021. doi:10.36311/jhgd.v31.10691

FLYNN, E. et al. Maternal depressive symptoms and child behavior among Mexican women and their children. International Journal of Environmental Research and Public Health, Suíça, v. 14, n.12, p. 1-14, 2017.

GABBARD, C.; KREBS, R. J. Studying environmental influence on motor development in children. The physical educator, EUA, $\mathrm{V}$. 69, n. 1, p. 136-149, 2012.

GIORDANI, L. G.; ALMEIDA, C. S.; PACHECO, A.M. Avaliação das oportunidades de desenvolvimento motor na habitação familiar de crianças entre 18 e 42 meses. Motricidade, Portugal, v. 9, n. 3, p. 96-104, 2013.

GOTTLIEB, G. Environmental and behavioral influences on gene activity. Current Directions in Psychological Science, EUA, v. 9, p. 93-97, 2000.

GREGOVISKI, V. R.; SILVA, F. L. L.; HLAVAC, L. A. B. 'É menino ou menina?' - a construção da identidade de gênero através dos 
Oportunidades de estimulação motora em ambientes domiciliares do Amazonas e fatores... Marcelo Gonçalves Duarte • Giseli Santos Dalpiaz Duarte • Glauber Carvalho Nobre • Thábata Viviane Brandão Gomes • Rodolfo Novellino Benda

brinquedos. Perspectiva, Florianópolis, v. 40, n. 152, p. 89-99, 2016.

INSTITUTO BRASILEIRO DE GEOGRAFIA E ESTATÍSTICA. Informações sobre os municípios brasileiros. Brasília.[s.n.]. Disponível em: http://www.cidades.ibge.gov.br/xtras/perfil. php?lang=\&codmun=130340\&search=amazonas\%7Cparintins\%7Cinfograficos:-informacoes-completas. Acesso em: 5 Jun. 2017.

JONES, P. C., et al. Measuring home environments across cultures: Invariance of the HOME scale across eight international sites from the MAL-ED. Journal of school Psychology, EUA, v. 64, oct., p. 109-127, 2017.

KOSHY, B. et al. Home environment: Short-term trends and predictors in early childhood from an Indian community birth cohort Home environment: Short-term trends and predictors in early childhood from an Indian community birth cohort. Child Care Health Development, London, v. 47, n. 8, p. 336-348, 2021.

LEWONTIN, R. The triple helix: gene, organism and environment. Cambridge: Harvard University Press, 2000.

MAROCO, J. Análise Estatística com o SPSS Statistics. 7. ed. Pêro Pinheiro: Report Number, 2018. 1013 p.

MÜLLER, A. B.; VALENTINI, N. C.; BANDEIRA, P. F. R. Affordances in the home environment for motor development: Validity and reliability for the use in daycare setting. Infant Behavior Development, EUA, v. 47, may, p. 138-45, 2017. doi: 10.1016/j. infbeh.2017.03.008

NASCIMENTO JUNIOR, J. R. A. et al. Nível socioeconômico e affordances do ambiente domiciliar: implicações para o desempenho motor infantil. Revista da Educação Física/UEM, Florianópolis, v. 25, n. 4, p. 651-662, 2014.

NOBRE, F. S. S. et al. Análise das oportunidades para o desenvolvimento motor (affordances) em ambientes domésticos no Ceará - Brasil. Revista Brasileira Crescimento e Desenvolvimento Humano, Santo André, v. 19, n. 1, p. 9-18, 2009. 
Oportunidades de estimulação motora em ambientes domiciliares do Amazonas e fatores... Marcelo Gonçalves Duarte • Giseli Santos Dalpiaz Duarte • Glauber Carvalho Nobre • Thábata Viviane Brandão Gomes • Rodolfo Novellino Benda

NOBRE, F. S. S. et al. Affordances em ambientes domésticos e desenvolvimento motor de pré-escolares. Pensar a Prática, Goiânia, v. 15, n. 3, p. 652-668, 2012.

PEREIRA, K. R. G.; VALENTINI, N. C.; SACCANI, R. Brazilian infant motor and cognitive development: Longitudinal influence of risk factors. Pediatrics International, Japan, v. 58, n. 12, p. 1297-306, 2016. doi: 10.1111/ped.13021

PILATTI, l. et al. Oportunidades para o desenvolvimento motor infantil em ambientes domésticos. Revista Brasileira de Ciências da Saúde, João Pessoa, v. 9, n. 27, p. 23-27, 2011.

PRADO, E. L.; DEWEY, K. G. Nutrition and brain development in early life. Nutrition reviews, Oxford, v. 72, n.4, p. 267-284, 2014. PROGRAMA DAS NAÇÕES UNIDAS PARA O DESENVOLVIMENTO [PNUD]. Desenvolvimento humano nas macrorregiões brasileiras. Brasília: Programa das Nações Unidas para o Desenvolvimento, 2016.

RODRIGUES, L. P. Projetc AHEMD: Affordances in the home environment for motor development, 2005. Disponível em: http:// www.ese.ipvc.pt/dmh/AHEMD/pt/ahemd_4pt.htm. Acesso em: 10 Jan. 2017.

RODRIGUES, L. P.; GABBARD, C. Avaliação das oportunidades de estimulação motora presentes na casa familiar: projecto affordances in the home environment for motor development. In: BARREIROS, J.; CORDOVIL, R.; CARVALHEIRA, S. (Eds.).

Desenvolvimento motor da criança. Lisboa: Edições FMS, 2007. p. 51-60.

RODRIGUES, L. P.; SARAIVA, L.; GABBARD, C. Development and Construct Validation of an Inventory for Assessing the Home Environment for Motor Development. Research Quarterly for Exercise and Sport, EUA, v. 76, n. 2, p. 140-148, 2005.

RODRIGUES, O. M. P. R.; NOGUEIRA, S. C.; ALTAFIM, E. R. P. Práticas parentais maternas e a influência de variáveis familiares e do bebê. Pensando famílias, Porto Alegre, v. 17, n. 2, p. 71-83, 2013. 
Oportunidades de estimulação motora em ambientes domiciliares do Amazonas e fatores... Marcelo Gonçalves Duarte • Giseli Santos Dalpiaz Duarte • Glauber Carvalho Nobre • Thábata Viviane Brandão Gomes • Rodolfo Novellino Benda

SILVA, J. et al. Pacing opportunities at home and skill of children with potential changes in functional development. Journal of Human Growth and Development, Santo André, v. 25, n. 1, p. 19-26, 2015.

SILVA, W. R. et al. Oportunidades de estimulação motora no ambiente domiciliar de crianças. Journal Human Growth Development, Santo André, v. 27, n. 1, p. 84-90, 2017. doi: http:// dx.doi.org/10.7322/jhgd.127659.

SOARES, E. S. et al. Análise das oportunidades de estimulação motora em ambientes domiciliares na região central do Rio Grande do Sul. Revista Brasileira de Educação Física e Esporte, São Paulo, v. 29, n. 2, p. 279-288, 2015.

TANI, G. et al. Educação física escolar: fundamentos de uma abordagem desenvolvimentista. São Paulo: EPU: EDUSP, 1998.

TOTH, K. et al. Home learning environment across time: The role of early years HLE and background in predicting HLE at later ages. School Effectiveness and School Improvement Talor \& Francis, UK, v. 31, n.1, p.7-30, 2020.

VALADI, S.; GABBARD, C. The effect of affordances in the home environment on children's fine- and gross motor skills. Early Child Development and Care, EUA, v. 190, n. 8, p. 1225-1232, 20 20. doi: 10.1080/03004430.2018.1526791

VIEIRA, M. T.; SILVA, J.; FRÔNIO, J. S. Functional capacity, independence and home affordances of premature children attending daycare centers. Fisioterapia e Movimento, Curitiba, v. 30, n. 1, p. 85-95, 2017. doi: http://dx.doi.org/10.1590/1980-5918.030.001. ao09

ZOGHI, A.; SHOJAEI, M.; GHASEMI, A. The impact of a motor affordance intervention on motor and cognitive development of young children. International Journal of Mental Health and Addiction, EUA, v. 14, n.5, p.743-750, 2016. 
Oportunidades de estimulação motora em ambientes domiciliares do Amazonas e fatores... Marcelo Gonçalves Duarte • Giseli Santos Dalpiaz Duarte • Glauber Carvalho Nobre • Thábata Viviane Brandão Gomes • Rodolfo Novellino Benda

\section{Publisher}

Universidade Federal de Goiás. Faculdade de Educação Física e Dança. Publicação no Portal de Periódicos UFG. As ideias expressadas neste artigo são de responsabilidade de seus autores, não representando, necessariamente, a opinião dos editores ou da universidade. 